\title{
Three-dimensional mapping of air flow at an urban canyon intersection
}

\author{
Matteo Carpentieri Alan G. Robins Sandro Baldi \\ Boundary-Layer Meteorology 133(2), 277-296 [2009] \\ doi: $10.1007 / \mathrm{s} 10546-009-9425-\mathrm{z}$
}

\begin{abstract}
In this experimental work both qualitative (flow visualisation) and quantitative (laser Doppler anemometry) methods were applied in a wind tunnel in order to describe the complex 3-dimensional flow field in a real environment (a street canyon intersection). The main aim was an examination of the mean flow, turbulence and flow pathlines characterising a complex 3-dimensional urban location. The experiments highlighted the complexity of the observed flows, particularly in the upwind region of the intersection. In this complex and realistic situation some details of the upwind flow, such as the presence of two tall towers, play an important role in defining the flow field within the intersection, particularly at roof level. This effect is likely to have a strong influence on the mass exchange mechanism between the canopy flow and the air aloft, and therefore the distribution of pollutants. This strong interaction between the flows inside and outside the urban canopy is currently neglected in most state-of-the-art local scale dispersion models.
\end{abstract}

\section{Introduction}

Air pollution in cities is a major environmental concern and, despite significant improvements in fuel and engine technology, present day urban atmospheric environments are mostly dominated by traffic emissions (Vardoulakis et al., 2003). Human exposure to hazardous substances is expected to be highest especially in those areas where population and traffic density are relatively high. Understanding flow and dispersion in urban streets is therefore of paramount importance for air quality management and planning for a number of reasons, mainly related to human health. Furthermore, the present international political situation adds further concerns, as the deliberate discharge of toxic material in populated areas is a serious threat.

The dispersion of pollutants and their causes and effects on buildings and population have been the object of a number of studies. However, those available in the literature generally deal with simplified layouts reproducing the principal features of an urban environment: canyons, intersections, regular and staggered building arrays. Empirical models have been developed along with numerical models and experimental validation in the field and in wind tunnels. Although most work has focussed on simple geometries, nevertheless some attempts to study real urban situations have produced interesting insights into the dispersion process.

The study of urban street canyons has dominated short range dispersion research in urban areas (see the reviews of Vardoulakis et al., 2003; Berkowicz et al., 1997; Britter and Hanna, 
2003). However, a number of studies (e.g. Soulhac, 2000, Scaperdas, 2000) showed the importance of three-dimensional effects at intersections, in particular the exchange of air, and hence pollutants, between the street systems involved. Pollution hotspots may be found at street intersections due to the presence of high traffic levels and traffic lights and they are more common than regular street canyons in real cities. Intersections show a highly complex flow that is strongly three-dimensional, as opposed to that in street canyons.

Most of the research in urban environment-related topics has primarily been concerned with concentration measurements. This is because this knowledge is directly linked with the assessment of effects on population exposure and health. Nevertheless, knowledge of the threedimensional flow field characterising a real urban environment is very important because it provides a better understanding of the possible paths followed by a pollutant released at urban, neighbourhood and local scales. Moreover the database produced by such an analysis is of great interest for the development of semi-empirical and numerical models, useful for predicting air quality in urban areas.

Pavageau et al. (2001) performed wind tunnel experiments on a two-dimensional canyon model set in a fetch of two-dimensional canyons that simulated the surrounding city and ensured full development of the internal boundary layer. Different aspect ratios, building heights, roof shapes and canyon lengths were considered. They pointed out a strong influence of canyon and roof geometry on wind-driven street ventilation. Xie et al. (2005) employed CFD techniques for the analysis of the influence of geometry on the mean flow field inside an urban canyon. A number of configurations was considered: (a) symmetrical canyon geometry; (b) step-up notch; (c) step-down notch. In the first case a single vortex was generated inside the canyon, while in the step-down notch case a vortex with its centre located above the lower roof level was found, with a second counter-rotating vortex located lower in the canyon. Finally, the step-up notch configuration presented a single distorted vortex with the centre of rotation slightly lifted towards the windward side. Garcia Sagrado et al. (2002) performed wind-tunnel experiments on both an isolated street canyon in open country and a non-isolated canyon. In both cases, a large separated flow region developed above the roof due to the approach flow impacting against the first upstream building. A recirculation region was observed above the street when the buildings forming the canyon were of the same height.

In the field experiments of Louka et al. (2000), mean and turbulent velocity fields were measured and the flow within and above a non-isolated street canyon studied when the wind blew perpendicularly to the street. The recirculation in the street was found to be unsteady and dominated by turbulent fluctuations. Furthermore they noted that the shear layer, shed from the upstream roof, became unstable through Kelvin-Helmholtz instability. The mixing of the air between the canyon and air aloft was therefore attributed to a large-scale flapping of the shear layer. Uehara et al. (2000) found that the flow in a street canyon was also strongly affected by atmospheric stability. In particular, the cavity eddy developing within the canyon became weaker when the atmosphere was stable and stronger when unstable. The mixing in the canyon was enhanced in unstable conditions, which caused the vertical temperature gradient to decrease and with it the thermal instability.

Britter and Hanna (2003), in a detailed review of flow and dispersion in urban areas, pointed out some features of flow in and around street canyons. The height of the buildings is a first discriminating parameter in defining flow regimes and affects the depth of the roughness sublayer, which is commonly identified as a region in which the underlying buildings lead to a spatial horizontal inhomogeneity of the flow and which is usually considered to extend to ap- 
proximately twice the building height. Some disagreement was found in the literature over the spatially averaged mean velocity profile over an urban canopy or very rough surface. These discrepancies were thought to be caused by the difficulty in specifying reference parameters, such as the friction velocity, displacement height or roughness length. This problem has also been partly described by Kastner-Klein et al. (2001). The geometry of the buildings and, in general, of the urban area also has a major influence on the production of turbulence inside and outside a canyon. Generally, for cities with large building plan and frontal area densities, turbulence is mainly generated by the interaction of the flow near the top of the canopy with the building tops and subsequent advection into the canyon. In small building plan and small frontal area density configurations, on the contrary, turbulence is generated within the canopy itself and is characterised by high Reynolds stresses in the region where turbulence is generated. As far as the recirculation flow within the canyon is concerned, this is neither steady nor symmetrical, with stronger and more concentrated downflow near the windward wall and a weaker and more extensive upflow closer to the leeward face. Large aspect-ratio configurations generally also contain a counter-rotating vortex below the main recirculation flow. Flow that is not perpendicular to the canyon generates a flow field consisting of a recirculation vortex and an along-street component, together forming a helical circulation. Finally, the role of traffic was identified as a further source of turbulence, together with wind and thermal gradients. The exchange mechanism in isolated and non-isolated canyons was part of the work by Meroney et al. (1996). They concluded that in a canyon in otherwise open country the vortex generated within the canyon itself is generally unstable and is discharged at regular intervals. On the contrary, inside an urban canyon a stable rotating vortex develops and street ventilation is suppressed, resulting in pollution being trapped at street level. Despite these studies, details of the exchange mechanisms, and the velocities and fluxes between a canyon and the flow above, are not well understood and stand in need of further research.

Kastner-Klein et al. (2001) underlined a common difficulty encountered in many studies. Observations from field measurements can rarely be compared with data from either wind tunnel or computational approaches. This is due, as has been pointed out above, to the inappropriate or unsatisfactory choice of a reference velocity and to the inherent uncertainties involved (see also Schatzmann et al., 1997). Nevertheless, their results are in substantial agreement with other studies and show that the flow over a street canyon is accelerated above roof level. Turbulence intensities were found to take relatively high values in the canopy layer above the canyon but rather uniform and low values within the canyon.

As is clear from the literature, the most important limit to the development of urban air quality models is the lack of experimental data. In the past there has been a tendency to perform rather narrowly focussed urban dispersion experiments (Robins and Macdonald, 2001), though several large field and wind tunnel experiments have recently been, or are being, attempted (Hunt et al., 2002). Physical modelling has been performed mostly within highly idealised geometries. As stated by Robins and Macdonald (2001), there is a need for tests in less 'regular' and more realistic building arrangements in order to produce more reliable datasets.

In the present work both qualitative and quantitative methods were used in order to describe the complex three-dimensional flow field in a real environment. Firstly, flow visualisation techniques were applied in a wind tunnel on a 1:200 scale model of a central London site, with particular focus on a busy street-canyon intersection. Following this qualitative description, a detailed mapping of the velocity flow field was performed using a two-component LaserDoppler Anemometer (LDA) to obtain velocity measurements on the same model. The main 
aim of the study was an examination of the mean flow, turbulence and flow pathlines characterising a complex, three-dimensional urban location.

The study is part of a multidisciplinary project, Dispersion of Air Pollution and its Penetration into the Local Environment (DAPPLE, Arnold et al., 2004), whose aim is to enhance understanding of pollutant dispersion processes in realistic urban environments. One of the novel aspects of DAPPLE, when compared to other similar studies, is its multidisciplinary approach to the problem: field measurements of wind conditions, background pollution levels, traffic flow, personal exposure and the dispersion of inert tracer releases were supported by both wind tunnel and numerical studies. Furthermore, the focus was a real urban intersection, characterised by buildings of different shape and height, not uninterrupted, two-dimensional canyons of different widths and lengths.

\section{Experimental set-up}

The DAPPLE site is located at the intersection of Marylebone Road and Gloucester Place in central London, U.K., with a surrounding study area approximately $250-300 \mathrm{~m}$ in radius. Wind tunnel modelling extends to a radius of about $500 \mathrm{~m}$. Marylebone Road is a busy dual carriageway (A501), up to seven lanes wide, and forms the northern boundary of the London Congestion Charging Zone, while Gloucester Place has three lanes, one-way northbound (Baker Street is southbound one block to the east). The roads intersect perpendicularly and Marylebone Road runs approximately from west-south-west to east-north-east. The prevailing wind is from south-west, and the average building height is approximately $22 \mathrm{~m}$.

The primary characteristic of the area is that it is a real site and the heights and sizes of the buildings and streets are all different (see figure 1); e.g. Marylebone Road is about twice as wide as Gloucester Place. Building 5, that is the Westminster City Council (WCC), is about 15 $\mathrm{m}$ tall, only $4 \mathrm{~m}$ taller than building 2 (Marathon House, $\mathrm{MH}$ ), which is the shortest. However, these are the heights to roof level and the common feature of the two buildings is a tower. The WCC tower has a small cross-section and is $34 \mathrm{~m}$ high whereas the tower on Marathon House is $53 \mathrm{~m}$ high, and has a wide cross-section. Both significantly affect the flow.

The experiments were carried out in the boundary layer wind tunnel of the Environmental Flow Research Centre (EnFlo), University of Surrey, U.K. This is an open circuit 'suck-down' wind tunnel with a $20 \mathrm{~m}$ long, $3.5 \mathrm{~m}$ wide and $1.5 \mathrm{~m}$ high working section. The air speed range is from 0.3 to $3.5 \mathrm{~m} \mathrm{~s}^{-1}$, and the facility is capable of simulating both stable and unstable atmospheric conditions, although this feature was not used in our study. Reference flow conditions are measured by two ultrasonic anemometers, one held at a fixed location and the other positioned as required, with two propeller anemometers mounted on either side of the traverse carriage. The motor shaft speed is also monitored. Temperature conditions are monitored by thermocouple rakes in the flow and individual thermocouples in each tunnel wall panel. The pressure drop across the inlet is also monitored, primarily to indicate the state of the inlet screens. The wind tunnel and the associated instrumentation are fully automated and controlled using virtual instrument software developed at EnFlo using LabVIEW.

A site co-ordinate system was defined to be aligned with the street network. The origin was the centre of the intersection, with the $X$ axis along Marylebone Road to the east. A wind direction of $0^{\circ}$ corresponded to flow parallel to the $X$ axis (in the west to east direction) and the model rotation angle was defined positive anticlockwise. 


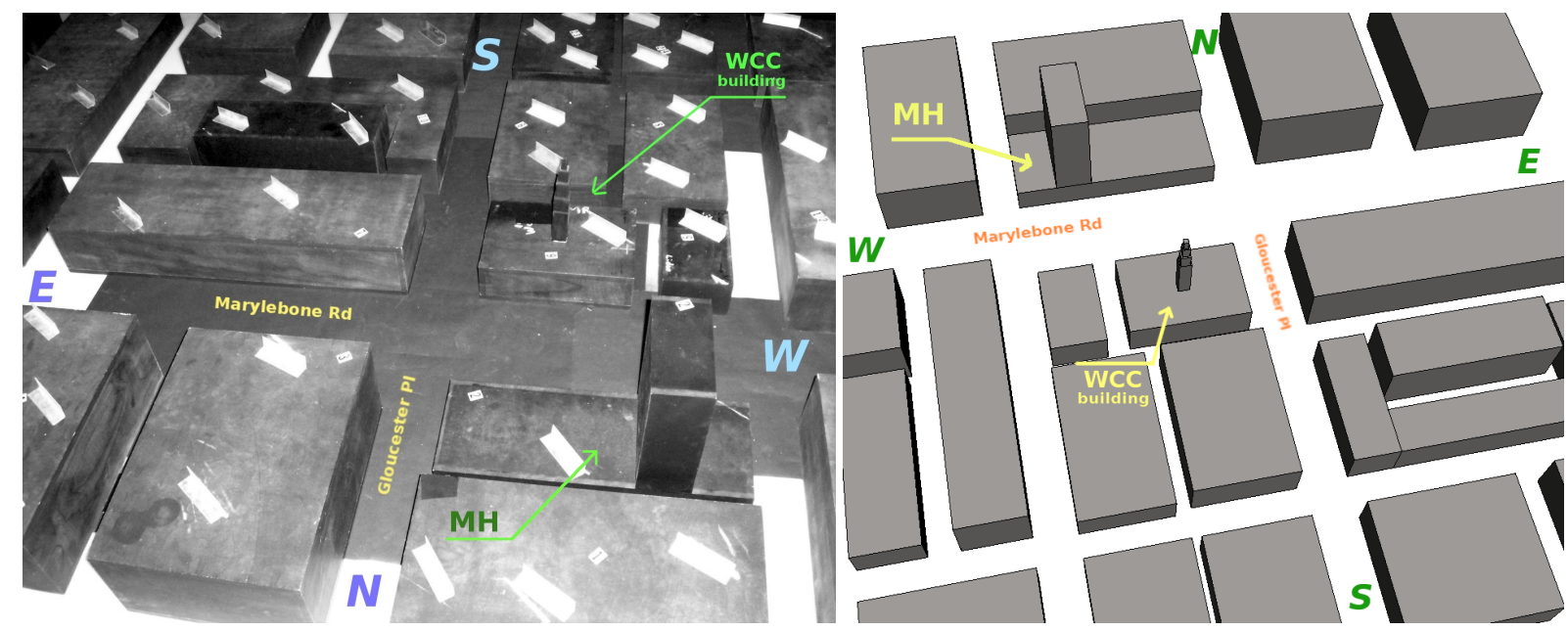

Figure 1: The 1:200 basic block model (left) and three-dimensional rendering (right) of the DAPPLE site in the EnFlo wind tunnel (WCC, Westminster City Council; MH, Marathon House)

The central part of the model installed in the wind tunnel is shown in figure 1-left. This is the simplest DAPPLE site model, where all buildings have been reduced to simple blocks with flat roofs at a geometrical scaling factor of 1:200. The approach flow boundary layer was generated in a standard manner, using Irwin spires and surface roughness upwind of the model. This produced a well developed boundary layer upstream of the model with thickness of approximately $1 \mathrm{~m}$, a surface roughness length $z_{0}=1.5 \mathrm{~mm}$ (equivalent to $0.3 \mathrm{~m}$ at full scale) and a normalised friction velocity, $u_{*} / U_{r e f}=0.057$. Details of this flow are included with the full experimental dataset, as described in table 1. Most of the tests were carried out with a reference air speed $\left(U_{r e f}\right)$ of about $2.5 \mathrm{~m} \mathrm{~s}^{-1}$. $U_{\text {ref }}$ was measured with the fixed ultrasonic anemometer positioned outside of the boundary layer. The model was oriented using the wind tunnel turntable, and all the experiments were performed with a rotation of $-51.35^{\circ}$ in model coordinates (i.e. wind approximately from the south-west); this wind direction was chosen as it corresponded to the direction for the first field tracer release experiment (Arnold et al., 2004).

Flow visualisation techniques were applied in order to describe qualitatively the flow patterns within the model. This work was then completed by high resolution measurements of wind velocity and turbulence, performed using a two-component laser Doppler anemometer.

\section{Flow visualisation}

\subsection{Experimental strategy}

Flow visualisation (FV) techniques were employed to analyse the flow field in an area in and around the Marylebone Road/Gloucester Place intersection using smoke that was released from a small source and illuminated by a laser light sheet. Light used to illuminate the smoke becomes scattered by smoke particles in all directions, and the intensity of scattered light at any position in the flow is, ideally, proportional to the concentration of smoke particles.

Video records were taken for each combination of source position and light sheet configuration. In order to obtain a detailed map of the flow at the intersection, a small smoke source was 
located in 12 different positions: at the centre, at the south side and at the north side of Marylebone Road (upwind from the intersection), and at the centre, at the east side and at the west side of Gloucester Place; further, sources were located at two different heights (10 and $40 \mathrm{~mm}$ ). A second set of experiments was subsequently carried out later, using the same experimental set-up. Smoke was released at five different positions (three in Marylebone Road, and two in Gloucester Pl), and at only one height $(10 \mathrm{~mm})$. The laser sheet was directed either westbound along Marylebone Road, or southbound along Gloucester Place. Horizontal sections were visualised at different heights (20, 40 and $60 \mathrm{~mm}$ during the first set of experiments, 25 and 50 $\mathrm{mm}$ during the second set). Three vertical sections in Marylebone Road (south side, centre, and north side) and one in Gloucester Place (centre) were also visualised during the experiments.

The video records produced by the flow visualisation experiments were then reviewed and analysed. Screenshots were also captured in order to highlight the main features of the observed flow.

\subsection{Flow in horizontal planes}

The main patterns of the observed horizontal flow field are shown in figure 2 (stills captured from the video records). The video records were essential in developing the flow descriptions and the stills in the figure are included here to illustrate specific features.

As far as the lowest levels $(20-25 \mathrm{~mm})$ are concerned, a large recirculating vortex was observed downwind of the intersection, at the south side of Marylebone Road (figure 2, top-left). In fact, the main tendency of the flow within Gloucester Place was to turn into Marylebone Road, continuing as a jet along the north side of Marylebone Road, and creating the recirculation vortex cited above. At higher levels, part of the flow from Gloucester Place continues along its path northwards. Intermittently however, the fraction flow affected by this behaviour increases, creating a smaller localised recirculation pattern in Marylebone Road upwind of the intersection (figure 2, top-right).

The incoming flow along Marylebone Road followed a more complex path. A highly turbulent flow was observed approaching the intersection, probably reflecting an underlying complex three-dimensional mean flow. At the intersection the general behaviour was to continue along Marylebone Road, joining the incoming jet from Gloucester Place. Just as for the latter, the flow occasionally deviated into Gloucester Place, causing another recirculation vortex on the west side of the street. Flow patterns were similar at higher levels, $40 \mathrm{~mm}$ and above, the only observable difference being the reduced size of the vortices in Gloucester Place and upwind, in Marylebone Road. The latter was also shifted somewhat towards the centre of the intersection.

\subsection{Flow in vertical planes}

The observed flow patterns in a vertical section close to the centre of Marylebone Road showed a strong vertical motion on the upwind side of the intersection, confirming what had previously been deduced. However, vertical motion was much reduced from approximately the intersection centre on (see figure 2, bottom-left). Intermittent small vortices could be seen in proximity to the centre of the intersection. The vertical flows observed in the central section were also seen in the vertical section on the south side. The chief difference was that these were confined to a smaller region in the upwind area of the intersection. 

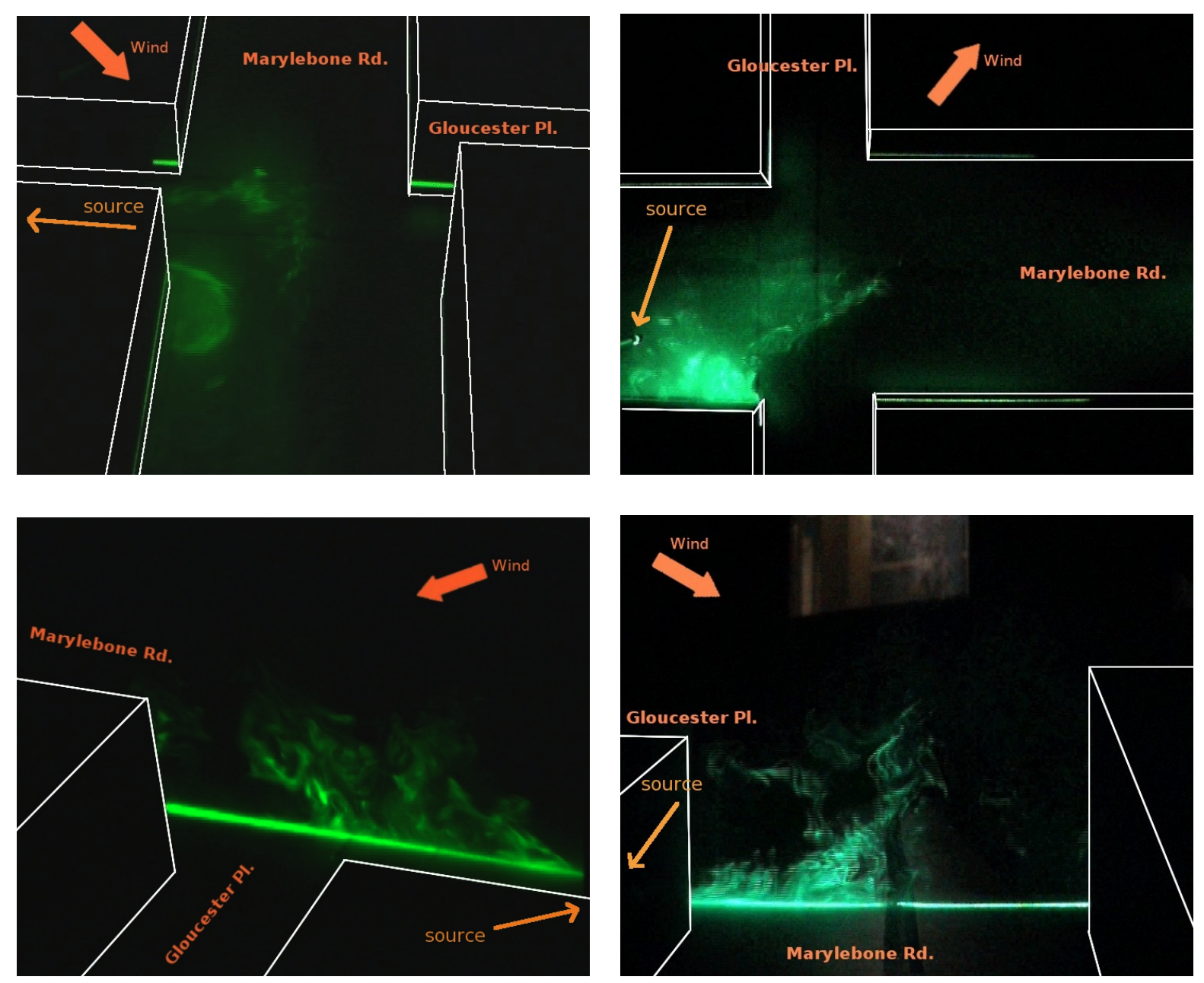

Figure 2: Still 1 (top-left): source at Gloucester Place (centre), horizontal light sheet at 20 $\mathrm{mm}$ in Marylebone Road showing vortex at the south-east corner of the intersection; still 2 (top-right): source at Marylebone Road (south), horizontal light sheet at $25 \mathrm{~mm}$ in Marylebone Road showing intermittent vortex at the south-west corner of the intersection; still 3 (bottomleft): source at Marylebone Road (centre), vertical light sheet in Marylebone Road (centre) showing the vertical motion of the smoke across the intersection and along Marylebone Road; still 4 (bottom-right); source at Gloucester Place (west), vertical light sheet in Gloucester Place (centre) showing the vertical motion of the smoke across the intersection 
In Gloucester Place (figure 2, bottom-right), unlike Marylebone Road, vertical motions were observed mainly in the downwind half or the intersection.

\subsection{Discussion}

Most of the analysis of the flow visualisation experiments was made by using the video recordings. The still pictures captured and presented here are useful for highlighting features of the flow, but most of the deductions made in the previous paragraphs, and in the following discussion, can be appreciated only by watching the videos. For this purpose, some selected video clips (see clips 1,2 and 3) have been provided as electronic supplementary material accompanying this article.

Analysis of the video recordings suggests that the flow inside the intersection can be divided in two different zones, one with a prevalence of near two-dimensional (horizontal) flow, and one with a more complex three-dimensional flow (localised mainly at the west and north-west sections of the intersection).

The main observed characteristics of the flow can be then summarised as follows:

- The main flow from Marylebone Road (west) is highly turbulent, three-dimensional and complex. It is mainly deflected upwards by the incoming flow from Gloucester Place, continuing into Marylebone Road (east), or deflected directly into Gloucester Place (north). For example, see clip 1 from the electronic supplementary material.

- Intermittently flow from Marylebone Road recirculates in a small vortex at the south-west corner of the intersection. This feature can be clearly seen in clip 1 as well.

- The flow from Gloucester Place is mostly entrained into Marylebone Road, forming a large recirculation vortex at the south side of the street. A significant fraction of the flow is deflected upwards and continues northbound at a higher level. See clips 1, 2 and 3.

- Intermittently the flow recirculates in the small horizontal vortex at the south-west corner of the intersection (see above) or vertically in vortices at the centre of the intersection. See clips 1 and 3.

The next objective was to move to quantitative measurements using laser Doppler anemometry.

\section{Laser Doppler Anemometry (LDA)}

\subsection{Experimental strategy}

Laser Doppler anemometry tests were performed in order to give a quantitative assessment of the observed flow within the Gloucester Place/Marylebone Road intersection. LDA is a non-intrusive optical method for measuring flow velocity based on the Doppler effect. The Doppler effect is the change of frequency observed whenever a source of light (or any type of wave) moves relative to a stationary observer. The change in frequency ('Doppler shift') is proportional to the velocity of the moving source. A fibre-optic LDA system was used, with the final optical head being a $20 \mathrm{~mm}$ diameter cylinder, $150 \mathrm{~mm}$ long and the measurement volume $50 \mathrm{~mm}$ in front of the head. 


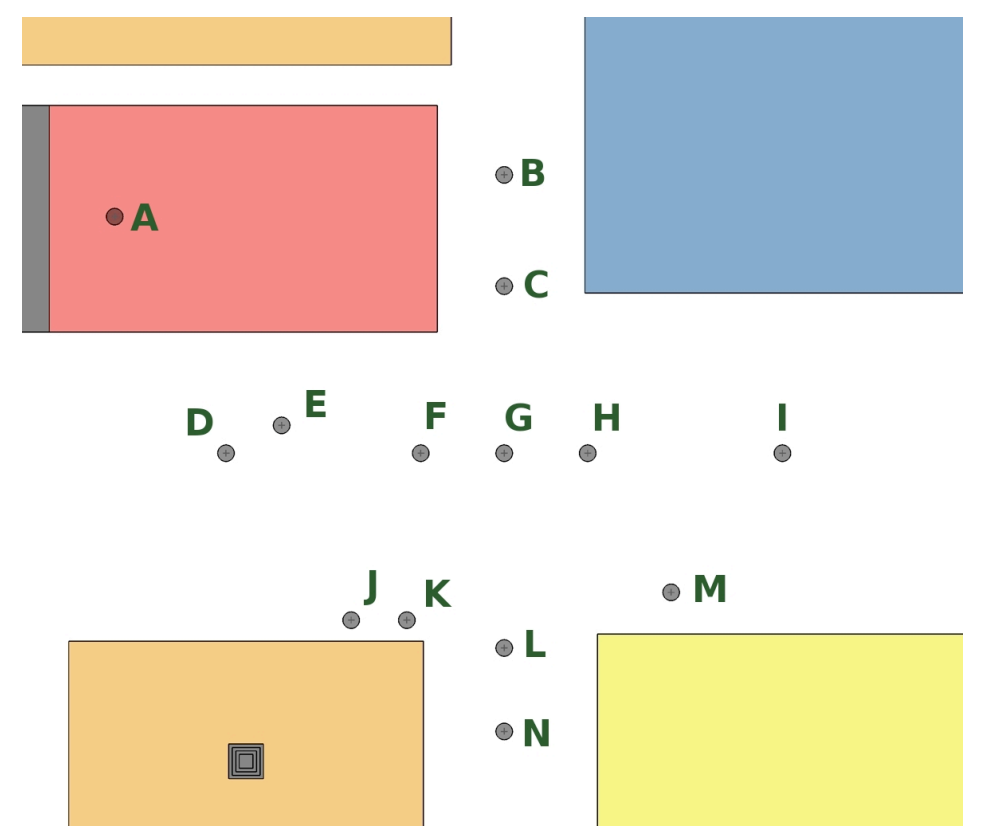

Figure 3: Locations of the three-minute average LDA vertical profiles

Three-dimensional LDA measurements were performed over the whole intersection. Considering the intersection as the origin of the coordinate system with the $x$ axis along Marylebone Road and the $Y$ axis along Gloucester Place, the area covered by measurements was the region $-300 \mathrm{~mm}<x<300 \mathrm{~mm},-300 \mathrm{~mm}<y<300 \mathrm{~mm}$ and $25 \mathrm{~mm}<z<150 \mathrm{~mm}$. The measurement grid consisted of $31 \times 31 \times 6$ points, spaced $20 \mathrm{~mm}$ along $x$ and $y$ and $25 \mathrm{~mm}$ along $z$.

In order to obtain the three velocity components over the whole area, the (head) probe of the two-component LDA system was employed in three different positions. Given the size and shape of the probe, some measurement positions in the street and close to the buildings were not feasible.

The three components of velocity were measured at almost all locations over a period of one minute for each point. The result is a complete three-dimensional mapping of the area. Mean and root-mean-square (rms) velocities, turbulent kinetic energy, vorticity, streamlines and Reynolds shear stresses were obtained from the analysis of the data. Furthermore, 14 points were chosen (see the map in figure 3) where velocity was measured along a vertical profile from $z=25 \mathrm{~mm}$ to $z=350 \mathrm{~mm}$ with an averaging time of 3 minutes. This became a more accurate evaluation of the velocity statistics by reducing the uncertainty in the 1 minuteaverage due to the low frequency fluctuations in the flow. Results from the LDA experiments are presented in non-dimensional form, using the reference wind tunnel speed, $U_{r e f}$, measured above the boundary layer as the velocity scale.

\subsection{Velocity and turbulence fields within the intersection}

Selected vector plots of the non-dimensional horizontal and vertical velocity fields are shown in figures 4 and 5 .

These results confirm findings from the flow visualisation experiments. In particular, the two main horizontal recirculation vortices can be clearly seen in Marylebone Road (south-east) 


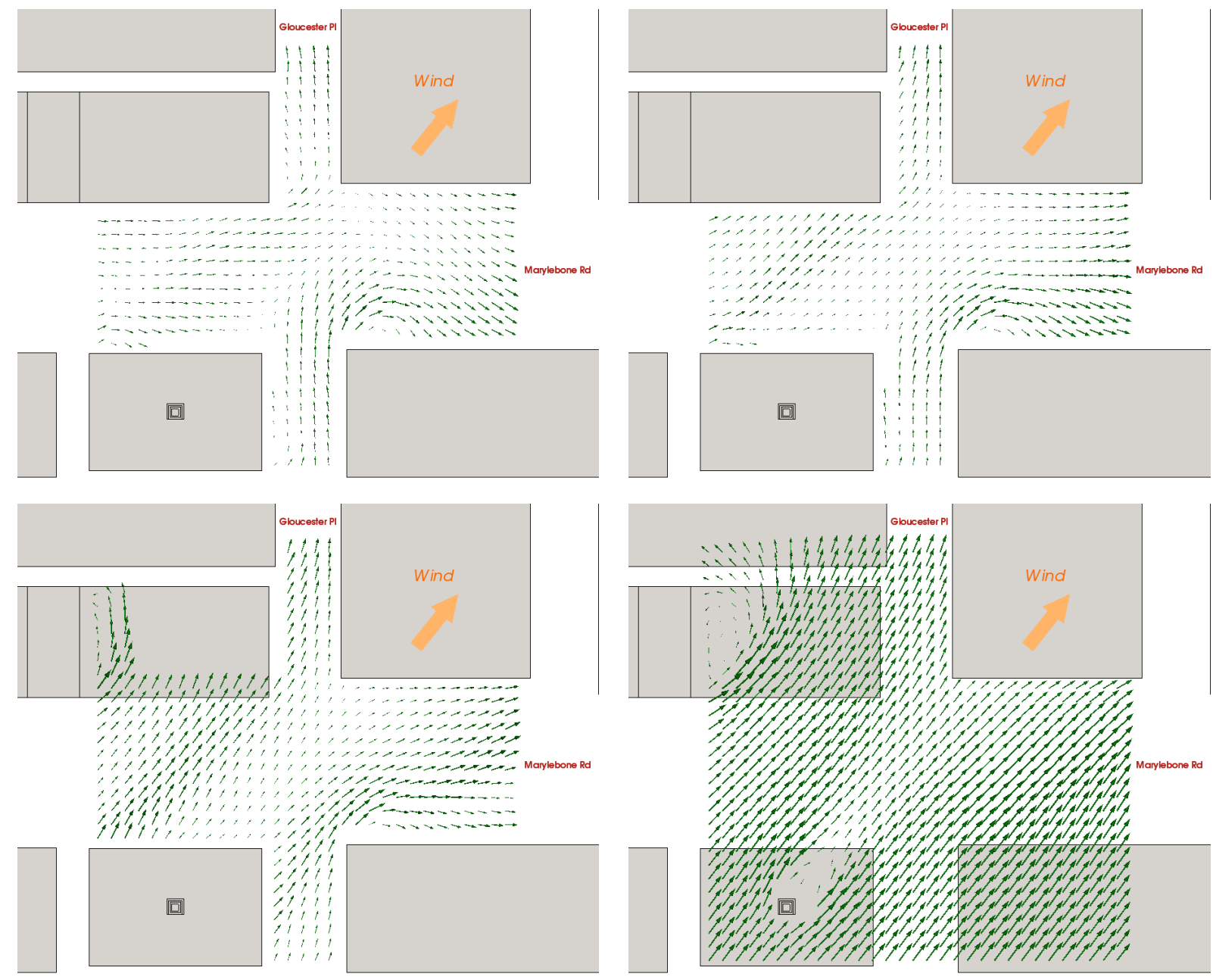

Figure 4: Horizontal velocity field at $z=25 \mathrm{~mm}$ (top-left), $z=50 \mathrm{~mm}$ (top-right), $z=75 \mathrm{~mm}$ (bottom-left) and $z=150 \mathrm{~mm}$ (bottom-right); mean building height $=110 \mathrm{~mm}$

and Gloucester Place (north-west). At a greater height, the flow and the turbulence field is increasingly dominated by the above roof wind and by the wake of the towers on the WCC building and Marathon House. Since the measures are averaged over one minute, they do not give reliable information about some intermittent characteristics observed in the flow visualisation experiments, such as the small recirculation vortex at the south-west corner of the intersection.

The most interesting characteristics of the vertical flow are shown in the Marylebone Road sections (e.g. see figure 5), because of its greater cross-section. Two main clockwise vortices can be observed in the sections upwind of the intersection (a smaller counter clockwise vortex may also exist but is not visible in the figures). Further downwind ( $x=260 \mathrm{~mm}$, approximately a street width from the intersection; see figure 5-bottom) the behaviour tends to be more like that of a classic street canyon flow field, with a large clockwise recirculation vortex.

The turbulent kinetic energy could be calculated at most measurements points, where all three velocity components were available. In a non-dimensional form, turbulent kinetic energy, 

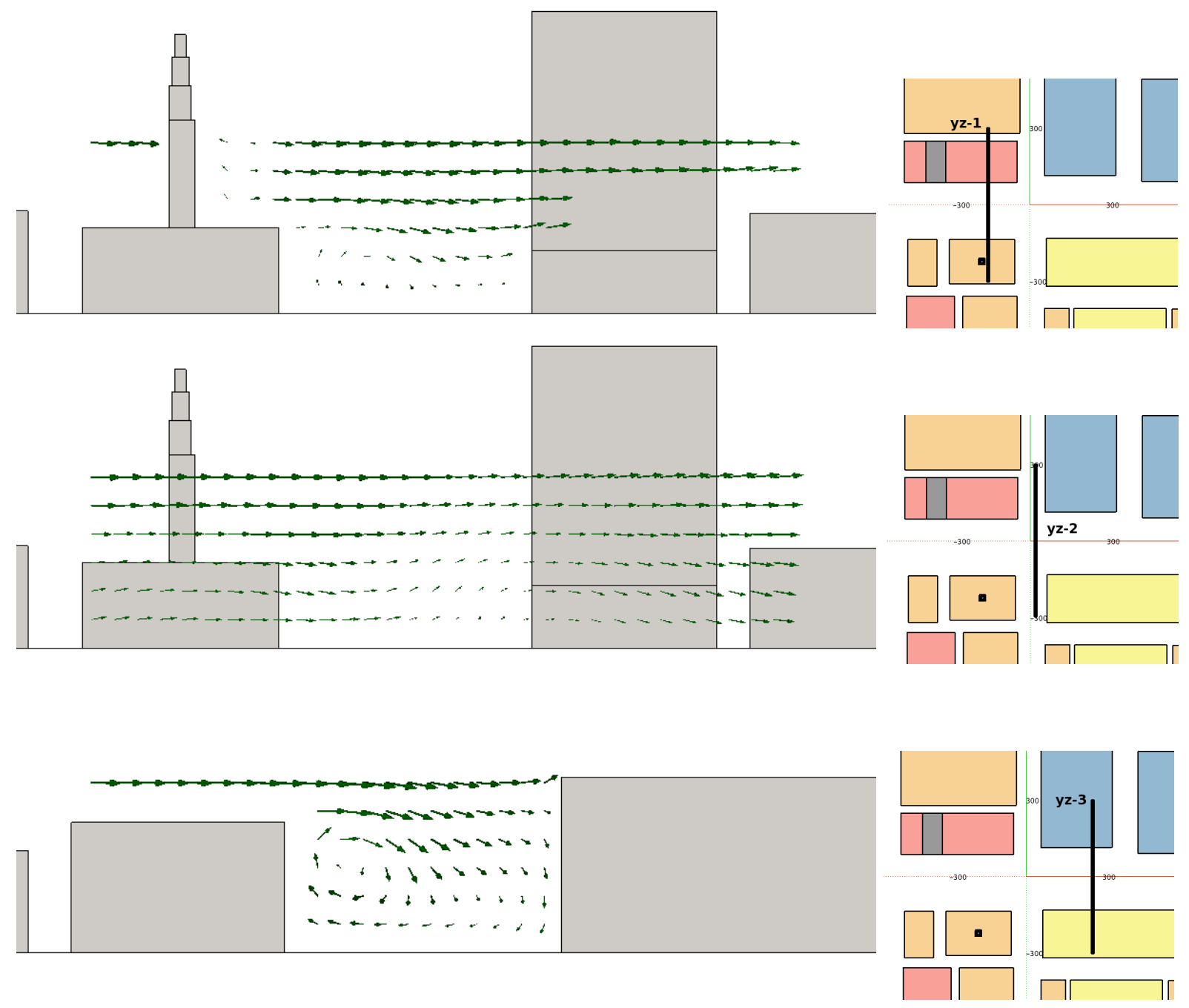

Figure 5: Vertical velocity fields in the $y-z$ plane; top: across Marylebone Road, west ( $x=$ $-160 \mathrm{~mm}$; “yz-1"); centre: along Gloucester Place ( $x=20 \mathrm{~mm}$; "yz-2"); bottom: across Marylebone Road, east ( $x=260$ mm; “yz-3”) 

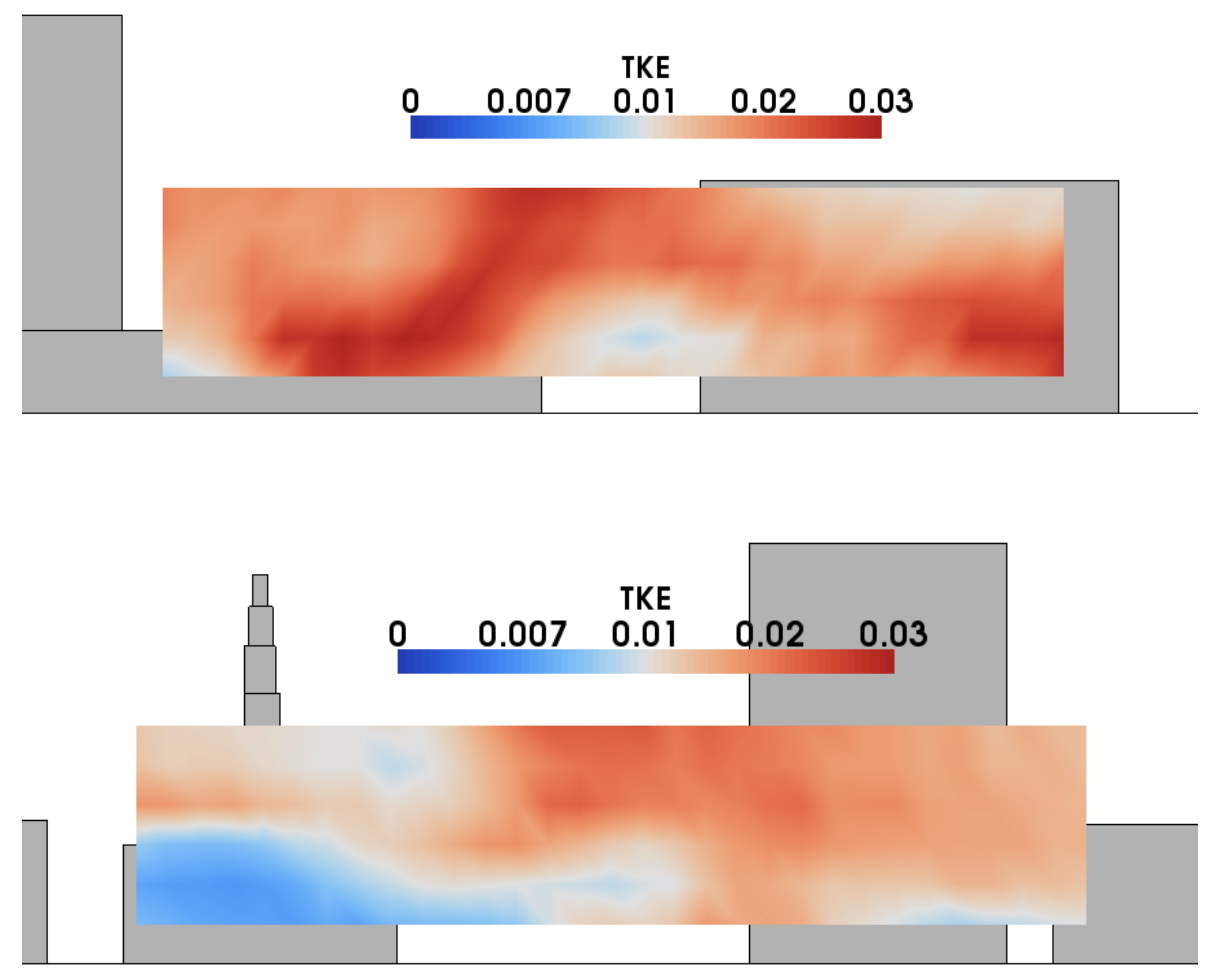

Figure 6: Contours of turbulent kinetic energy in vertical sections at $y=0 \mathrm{~mm}$ (top: $x-z$ plane, along Marylebone Road), and at $x=20 \mathrm{~mm}$ (bottom: $y-z$ plane, along Gloucester Place)

$e^{\prime}$, can be defined as:

$$
e^{\prime}=\frac{\left\langle u^{\prime}\right\rangle^{2}+\left\langle v^{\prime}\right\rangle^{2}+\left\langle w^{\prime}\right\rangle^{2}}{2}
$$

where $u^{\prime}=u / U_{\text {ref }}, v^{\prime}=v / U_{\text {ref }}, w^{\prime}=w / U_{\text {ref }}$ are the non-dimensional velocity fluctuations (i.e. the turbulent components) along, respectively, $x, y$ and $z$.

Some of the results are shown in figure 6.

Analysis of the figures for the lower levels reveals a highly turbulent area in Marylebone Road, upwind from the intersection, and also in the north-west corner of the intersection. A region of relatively low turbulence levels can be seen on the south-east side, with the position of the large recirculation vortex clearly visible. This behaviour is in full agreement with the flow visualisation. At higher levels, at and above roof level, the turbulence field is mainly influenced by the presence of the towers on the WCC and Marathon House buildings. Vertical sections (figure 6) show peaks in the turbulence levels located approximately at the building roof height. Since the building geometry is very heterogeneous, the height of these turbulence peaks is also very variable.

The construction of streamlines, or stream traces, provides a powerful analysis tool that is made possible by the availability of the three-dimensional flow measurements. The stream traces (derived by use of the open-source software ParaView 3.2.1) generally confirmed the previous analysis. The added capability of this form of analysis is the possibility to visualise three-dimensional patterns, as opposed to light sheet flow visualisation and LDA vector plots 


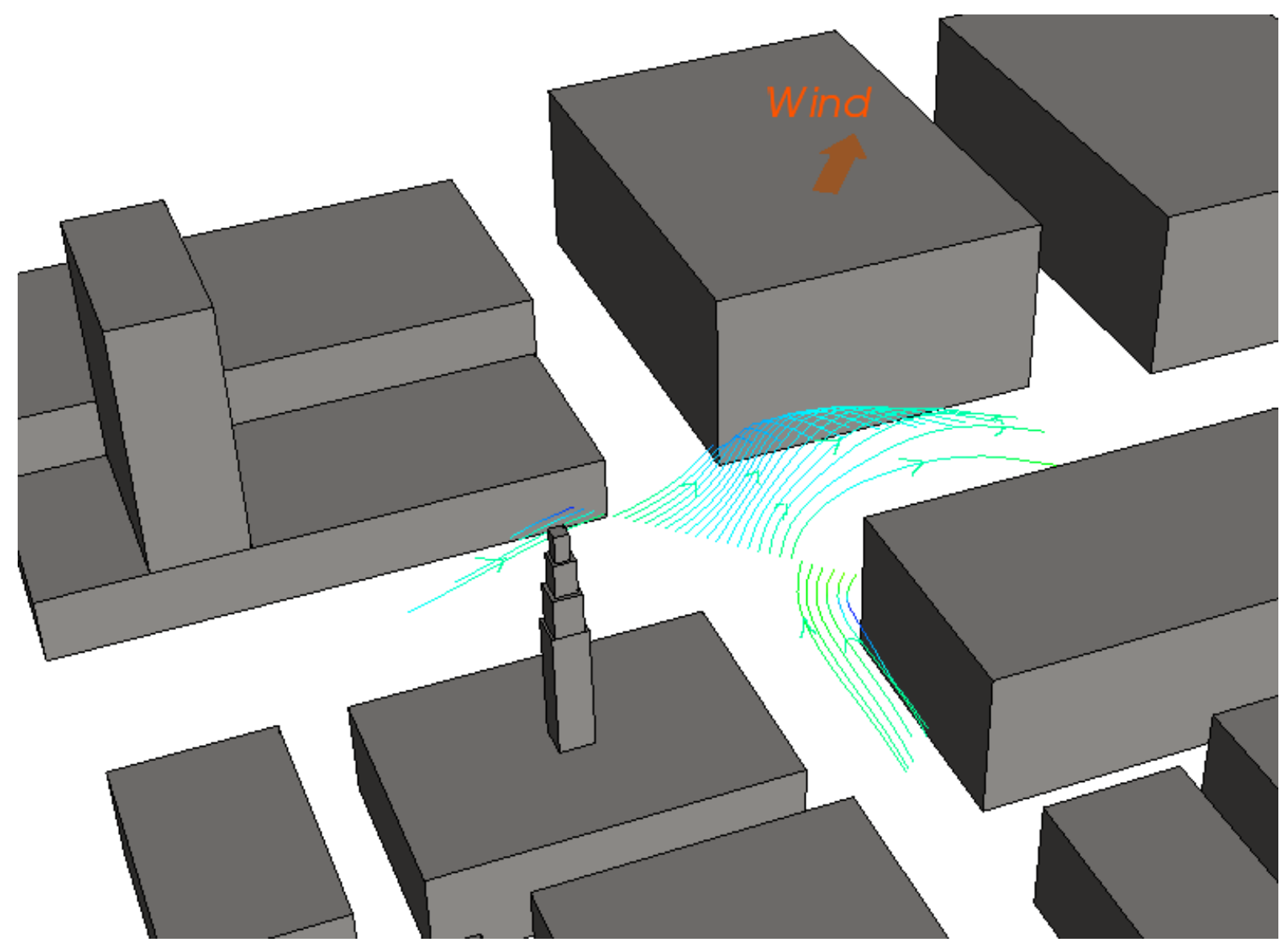

Figure 7: Stream traces for the flow field at the lowest levels. Starting points for both forward and backward traces are on the diagonal line from north-west to south-east across the intersection at $z=25 \mathrm{~mm}$. Traces terminate when outside of the measurement domain: $-300 \mathrm{~mm}$ $<x, y<300 \mathrm{~mm}, 25 \mathrm{~mm}<z<150 \mathrm{~mm}$

which give a two-dimensional view. This made it possible to highlight other interesting features of the studied flow (see figures 7, 8 ).

Figure 7 shows some interesting characteristics of the flow at the lowest levels by the termination of the traces. This implies that the flow is directed to, or comes from, areas beyond the boundaries of the measurement grid (e.g. the lowest level, at $25 \mathrm{~mm}$ in this case). Most of the flow at the intersection appears to be directed slightly upwards and, at this level, is mainly entrained into Marylebone Road, as already pointed out. Further from the intersection the streamlines are again directed downwards and the flow is most probably channelled into the street that runs parallel to Gloucester Place (Glentworth Street). On the other hand, the flow field near the north-west and south-east corners of the intersection is directed slightly downwards. This figure reveals a limitation of the LDA measurements, as the flow field below 25 $\mathrm{mm}$ ( $5 \mathrm{~m}$ at full scale) is completely unknown and this may greatly affect the possibility of performing accurate flux balances around the intersection (as this is a region of the flow where velocity and turbulence gradients are large).

Other characteristics are highlighted by figure 8. The substantial geometrical variability of this real urban intersection leads to a strong interaction between the canopy flow and the above-canopy flow. This is a quite different situation than that found with an ideal symmetric 


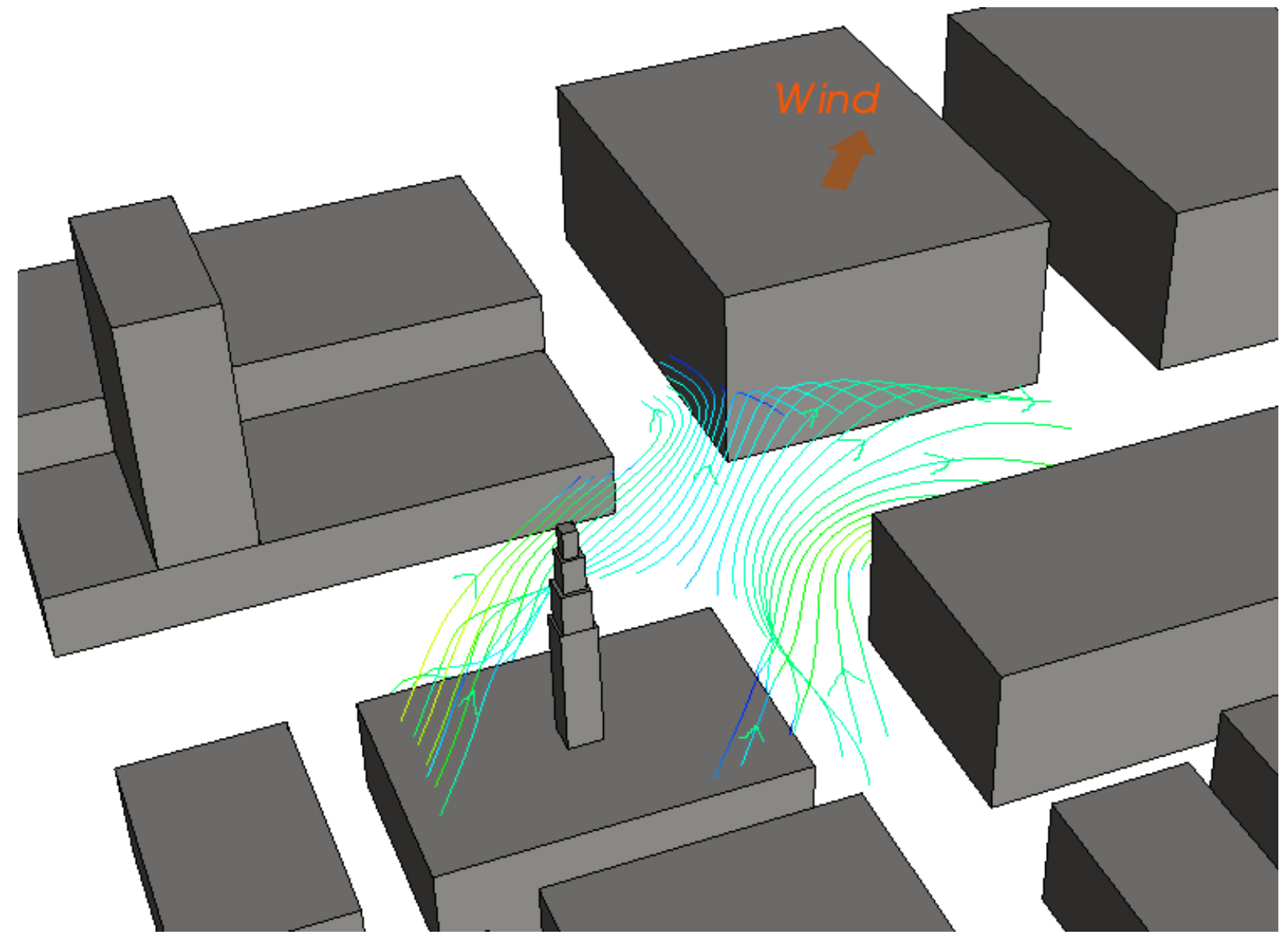

Figure 8: Stream traces for the flow field at the intermediate levels. Starting points for both forward and backward traces are on the diagonal line from north-west to south-east across the intersection at $z=50 \mathrm{~mm}$. Traces terminate when outside of the measurement domain: -300 $\mathrm{mm}<x, y<300 \mathrm{~mm}, 25 \mathrm{~mm}<z<150 \mathrm{~mm}$ ) 
canyon, where the above-canopy flow is often treated separately from the canyon flow.

Figures 9 and 10 present a selection of the results of the three-minute averaged measurements (see section 4.1 and figure 3) as vertical profiles at selected points along Marylebone Road and Gloucester Place. The profiles cover the mean and rms velocities and the Reynolds shear stresses. Near-classical street canyon behaviour can be seen at points in Gloucester Place upwind (at $\mathrm{L}$ and also $\mathrm{N}$, not reported in the figures) and downwind (at $\mathrm{C}$ and also $\mathrm{B}$, not reported in the figures) of the intersection, where the street canyon is narrower. However, the peaks in turbulence intensity at roof level are relatively weak because of the variable building heights. The influence of the buildings extends to higher levels than in simple geometries for the same reason. Similar behaviour can be also observed in the most easterly point in Marylebone Road (i.e. at point I). This point confirms, for this real situation, the findings of Xie et al. (2008) in the context of a generic randomised height geometry.

A more complex pattern is observed within the intersection and also in the upwind part of Marylebone Road. The influence of relatively tall upwind buildings can be seen in the vertical profiles. A 'double' adjustment in the vertical velocity profile is evident at points D and $\mathrm{H}$ in figure 10 and $\mathrm{G}$ in figure 9 (similar profiles were also obtained at points $\mathrm{E}$ and $\mathrm{F}$ ). The first adjustment level corresponds to the roof level of the WCC building $(75 \mathrm{~mm})$ and the second to the height of the WCC tower $(170 \mathrm{~mm})$. This is also clearly visible in the rms and Reynolds shear stress profiles, where a double peak can be observed and relatively high values are maintained from the roof level to the WCC tower height. This behaviour is even more marked at points K and J, (located in the wake of the WCC building) and analysed in the following section.

\subsection{Influence of tall buildings}

As highlighted by the analysis presented in the previous section, high-rise buildings exert a strong influence on the flow and turbulence fields in the vicinity of the street intersection and are areas where vertical exchanges might be concentrated. Figure 11 (top-left) shows a vector plot of the horizontal velocity field, together with a contour plot of the vertical velocity field, downwind of the tower on Marathon House.

The figure highlights the complex three-dimensional flow field in the wake of such a tower. In the region mapped the approach flow is directed downwards in much the near wake, but with some upward flow on the north side. In contrast, upwards flow dominates further downwind. The resulting $x$-vorticity component is shown in figure 11 (bottom), making clear the large scale swirling motion responsible for the pattern seen in figure 11 (top-right).

This feature of the flow is particularly strong near roof level, and it will clearly affect the vertical exchange mechanisms between the street canopy and the flow above roof level, a matter of great importance for dispersion models at this particular scale. As highlighted by figure 11 (top-right), a strong influence on the roof level flow is also exerted by the smaller tower on the WCC building, even though its effects seem to by mainly in the horizontal, particularly affecting the lateral mixing processes.

Further evidence on the strong influence of the taller buildings on the vertical exchange mechanisms can be found by analysing vertical profiles at points placed within the wake of the buildings and the towers (see figure 12).

The vertical profile at point $\mathrm{M}$ (in the wake of building 7 but with no taller upwind buildings) shows a large variation in mean velocity at a height that corresponds to the upwind roof level 
Point L
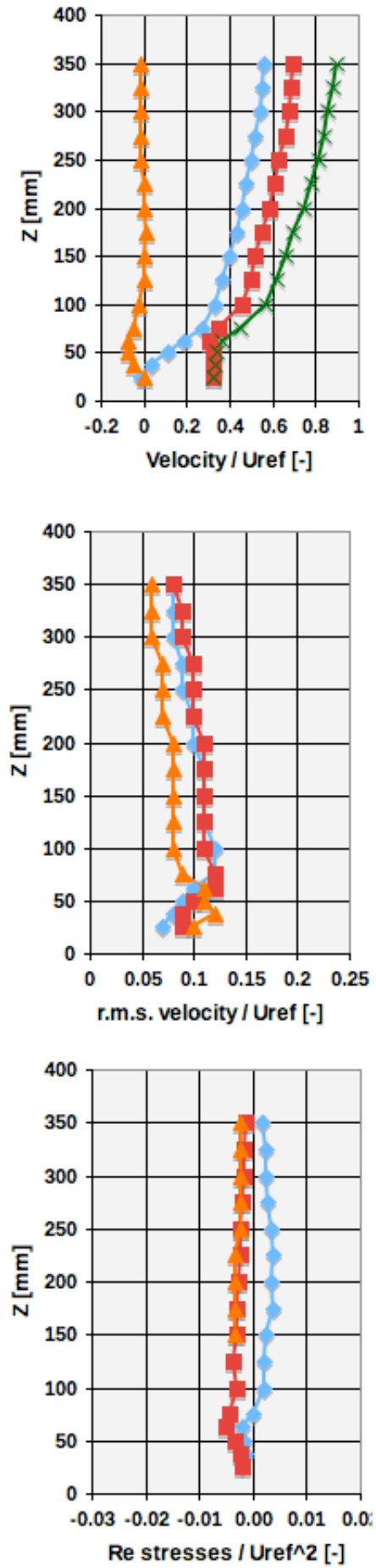

Point G
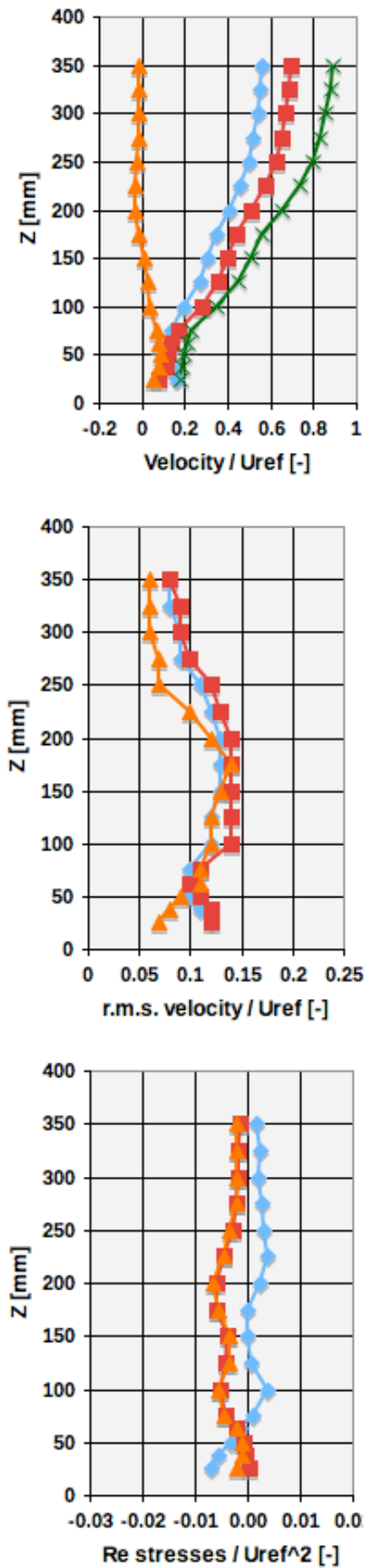

Point C

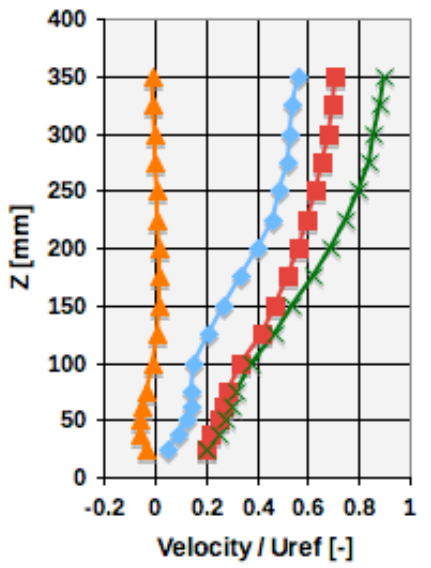

$\multimap$ U/Uref

- - VIUref

- WIUref

$\rightarrow$ Vtot/Uref

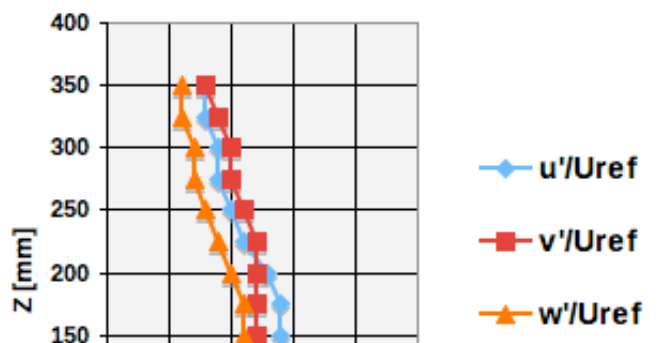

Figure 9: Vertical profiles of velocity (top), rms turbulence intensity (centre) and Reynolds shear stresses (bottom) along Gloucester Place (at points L, G and C shown in figure 3) 
Point D
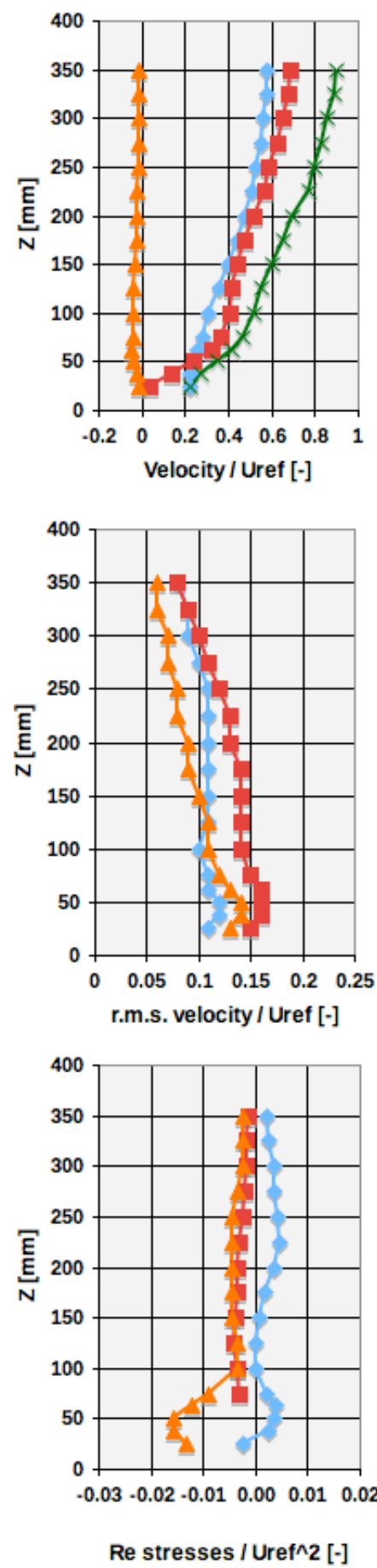

Point H
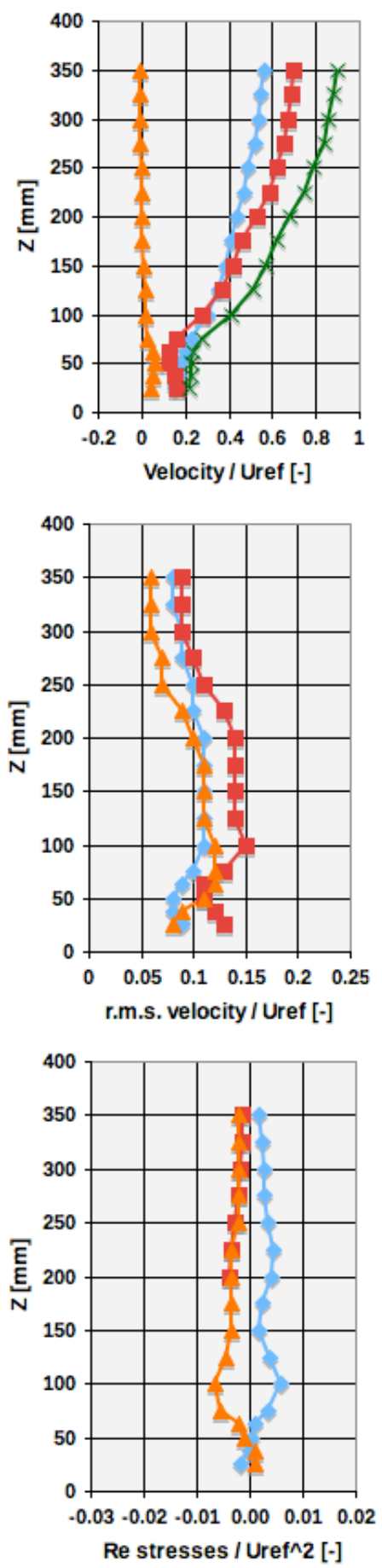

Point I
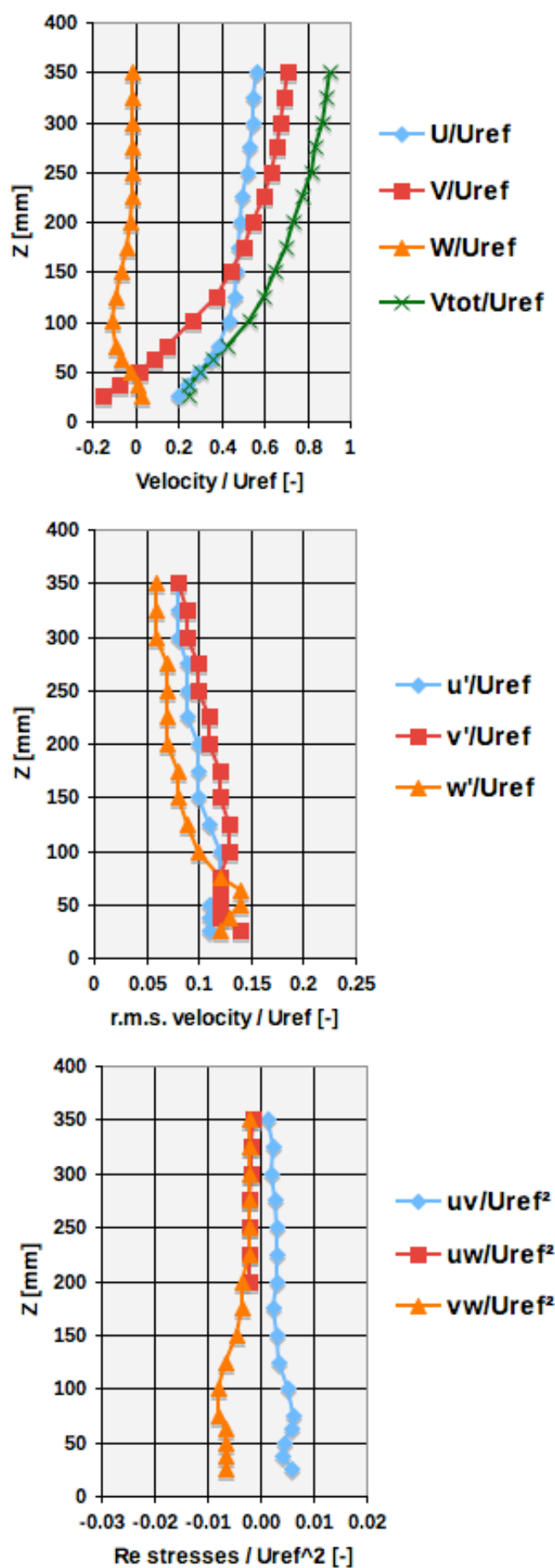

Figure 10: Vertical profiles of velocity (top), rms turbulence intensity (centre) and Reynolds shear stresses (bottom) along Marylebone Road (at points D, H and I shown in figure 3). Note that point $\mathrm{G}$, shown in figure 3 is part of the sequence 


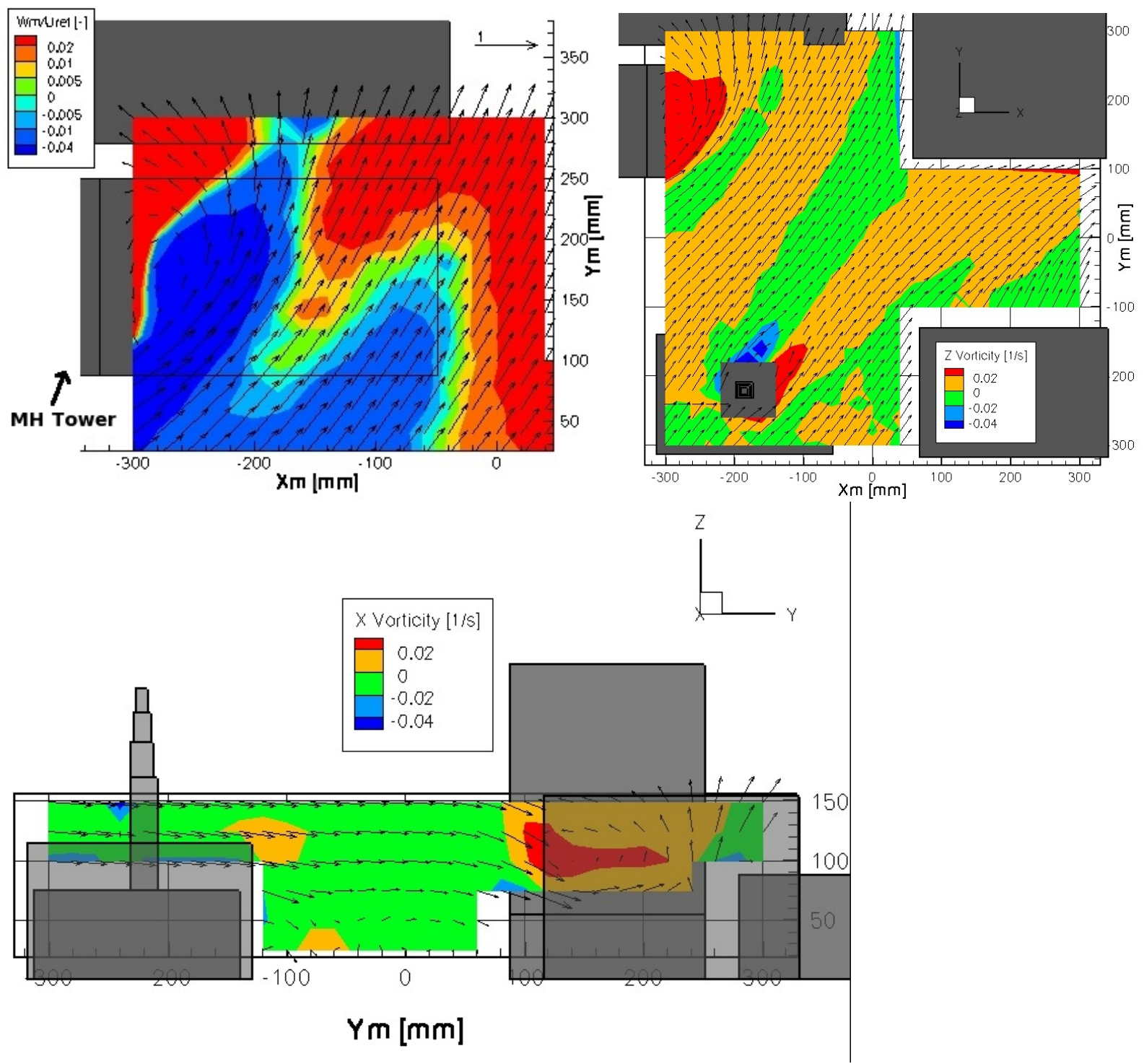

Figure 11: Top-left: vertical (contour plot) and horizontal (vector plot) flow fields in wake of the Marathon House tower at a height of $z=150 \mathrm{~mm}$; top-right: horizontal $(z)$ vorticity at $z=$ $125 \mathrm{~mm}$ (contour plot) and horizontal velocity field (vector plot); bottom: vertical ( $x$ ) vorticity at $x=-300 \mathrm{~mm}$ (contour plot) and vertical $(y-z)$ velocity field (vector plot) 
Point M
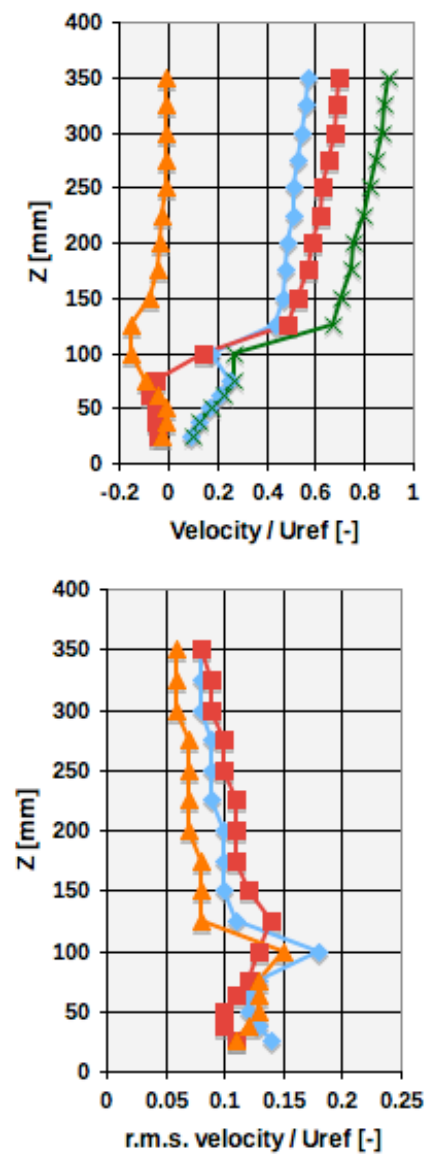

Point K
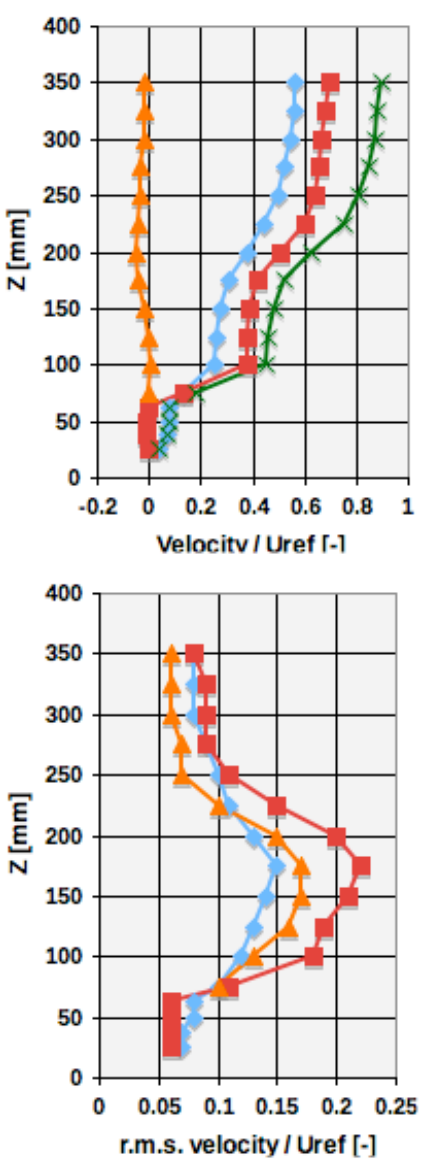

Point A

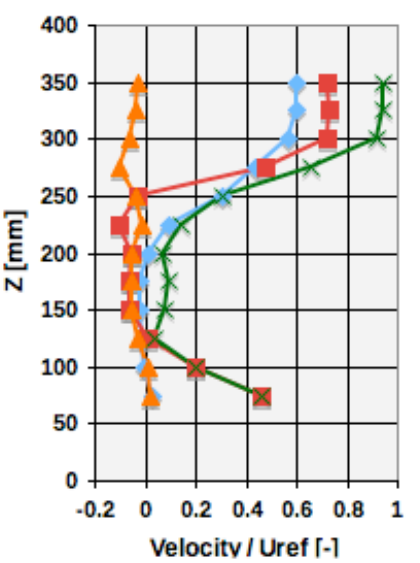

$\multimap$ U/Uref

- - VIUref

- WIUref

$\leftarrow$ Vtot/Uref

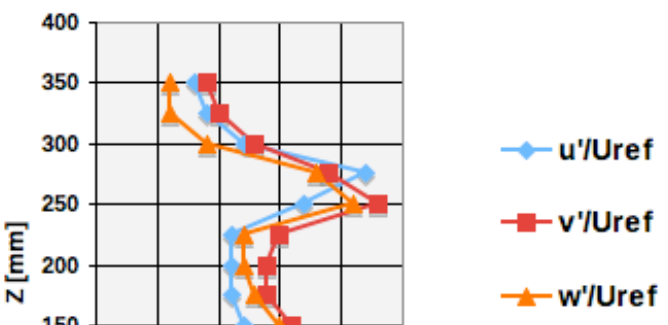

Figure 12: Vertical profiles in the wake zones (points M, K and A in figure 3); velocities (top) and rms (bottom) 
( $\sim 115 \mathrm{~mm})$. There is also a peak in the turbulence profiles at this height. Vertical exchanges are mostly confined to the shear layer at roof level. The situation of point $\mathrm{K}$, in the wake of both the WCC building and its tower, is substantially different. A double step is clearly visible in the vertical velocity profiles, corresponding to both the WCC building roof level and tower height, with turbulence levels remaining high throughout the whole layer between the two. Similar, though less pronounced behaviour is seen at points $\mathrm{G}, \mathrm{F}$ and $\mathrm{H}$ further downwind. The mean flow in the wake of the WCC tower tends to remain mostly horizontal, as observed previously, but all three turbulence components are relatively large. Point A reveals a slightly different behaviour. Again, the two turbulence peaks are observable in this case but the intensities are significantly reduced in the layer between the peaks. Clearly, the influence of the Marathon House tower at A can be characterised as a building near-wake, as opposed to the WCC tower which is best represented as creating a strong wake-like disturbance. The near-wake sheltering effect is clearly visible in the mean velocity profiles, in a substantial layer between the level of the Marathon House roof and tower top where velocities approach zero. Above the tower roof level, the vertical velocity profiles adjust again to match the external wind field, with a large local gradient.

\subsection{Discussion}

The LDA experiments clearly confirm that the 'average' characteristics observed by flow visualisation, although no further information about the intermittent behaviour previously identified has been obtained from the experiments.

The mean flow shows a rather complex behaviour across the whole area. Recirculation vortices were observed in a number different locations, though some were not that well defined and tended to merge into the along-street component of the flow. This is rather important because the wind is directed approximately $51^{\circ}$ relative to Marylebone road and therefore the $x$ and $y$ components are similar in magnitude. Generally, the flow in Gloucester Place near the intersection appears to be almost two-dimensional with an almost zero vertical component, although further from the intersection classical canyon vortex flows were identified. The patterns change in the region near and above roof level, as wakes and vortices from upwind structures interact quite strongly. The asymmetric configuration of the building geometry plays an important role in defining the mean and turbulent flow for $z>75 \mathrm{~mm}$ almost everywhere in the area. The mean flow along Marylebone Road is much more complex. Flow approaching the intersection from upwind $(x<0)$ in the lower part of the street canyon is generally deflected upwards in crossing the intersection, then downwards again, in part in Gloucester Place (north) and in part in Marylebone Road (east).

Measurements could not be taken closer to the surface than $z=25 \mathrm{~mm}$ but data from this level show significant downward components (i.e. towards the ground) in some areas and an upward components in others. This implies that the flow very close to the ground could be rather complex and that further measurements might well be needed (e.g. to enable flux balances to be calculated). The mean flow in Marylebone Road downwind of the intersection is fully three-dimensional, with vortices from the building on the lower-right corner of the intersection interacting with the down-flow coming from the upper levels of Marylebone Road (west). Nevertheless, in this region the recirculation vortices are clearly visible and the flow begins to assume a more "classical" canyon-style structure.

As observed in the flow visualisation experiments, this 'average' behaviour often breaks 
down. In such circumstances, a large part of the flow then passes northwards along Gloucester Place, creating some small recirculation regions at the west side of the intersection, both to the south of the intersection (in Marylebone Road) and to the north (in Gloucester Place).

The geometrical characteristics of the site (notably, towers and tall buildings) impose a very strong influence of the flow, causing the development of extensive recirculation areas, vertical exchanges and mixing in the wake. For example, the tower above the WCC building, despite its relatively small dimensions, exerts a strong influence over a large area of the intersection, mainly at $z>75 \mathrm{~mm}$.

The analysis of the Reynolds stresses shows that the boundary layer might be affected by the buildings up to heights of about $z=350 \mathrm{~mm}$, which is more than two times the height of the tallest building at the intersection. This effect is no doubt influenced by the two towers, though further analysis is needed. Nevertheless, the profiles show peaks at the level of the building roofs, which suggests that much of the turbulence is produced there.

\subsection{Quality assessment and datasets}

The LDA experiments were preceded by a series of preliminary measurements to optimise the experimental procedures; e.g. to determine the required averaging time. Preliminary runs and comparisons with a previous set of LDA data suggested that an ideal averaging time would be about 5 minutes. Nevertheless, as we were planning to perform a complete, detailed threedimensional mapping of the velocity field at the intersection, and considering the number of measurement points, it was decided to use one minute averages over the whole intersection and add some more accurate three minute averages as vertical profiles.

Error estimation was performed by using a 60-minute run of data, applying different averaging procedures and comparing subsequent results to the full 60-minute average. In this way, the error in one minute averages was found to be $\pm 9.6 \%$ for the $U$ component and $\pm 14.4 \%$ for the $V$ component; for three-minute averages, the corresponding errors were $\pm 3.2 \%$ and $\pm 5.9 \%$ and for five-minute averages $\pm 2.9 \%$ and $\pm 4.5 \%$.

Due to the long duration of the LDA experiments (each being several hours in duration), it was decided not to repeat any of the vertical or horizontal section measurements for the purpose of testing their repeatability. Instead, most of the measurement positions in the vertical sections (i.e. the $x-z$ or $y-z$ planes) were purposely located at the same points where horizontal section measurements were also made (i.e. in the $x-y$ plane) and these 'duplicate' measurements were then compared. The difference between such measures was within $\pm 10 \%$ for the great majority of points, and within $\pm 30 \%$ for nearly all cases. Of course, the fine spatial grid provided a further consistency check on the data.

\section{Conclusions}

Wind tunnel experiments were performed on a 1:200 scale model of a central London site, with particular focus on a busy street canyon intersection, in the framework of the DAPPLE project. Both qualitative flow visualisation techniques and LDA measurements were applied in order to describe the complex three-dimensional flow field in this real environment. The large experimental database that was compiled is summarised in table 1 . 
Table 1: Summary of the experiments performed and available data. The database also comprises full approach flow and geometry information

\begin{tabular}{|c|c|c|}
\hline Technique & Experiments & Description \\
\hline FV & $\begin{array}{l}\text { Horizontal sections in } \\
\text { Marylebone Road }\end{array}$ & $\begin{array}{l}\text { Video, laser sheet westwards, heights: } 20,40 \\
\text { and } 60 \mathrm{~mm}\end{array}$ \\
\hline FV & $\begin{array}{l}\text { Horizontal sections } \\
\text { Gloucester Place }\end{array}$ & $\begin{array}{l}\text { Video, laser sheet southwards, heights: } 20,40 \\
\text { and } 60 \mathrm{~mm}\end{array}$ \\
\hline FV & Vertical sections & $\begin{array}{l}\text { Video, laser sheet westwards in Marylebone } \\
\text { Road (centre, north and south sides) and south- } \\
\text { wards in Gloucester Place (centre) }\end{array}$ \\
\hline FV & Further horizontal sections & $\begin{array}{l}\text { Video, laser sheet westwards in Marylebone } \\
\text { Road, and southwards in Gloucester Place, } \\
\text { heights: } 25 \text { and } 50 \mathrm{~mm}\end{array}$ \\
\hline FV & Further vertical sections & $\begin{array}{l}\text { Video, laser sheet westwards in Marylebone } \\
\text { Road, and southwards (centre street) }\end{array}$ \\
\hline LDA & $\begin{array}{l}U-V \text { components measure- } \\
\text { ments }\end{array}$ & $\begin{array}{l}\text { Grid: }-300 \mathrm{~mm}<x<300 \mathrm{~mm} ;-300 \mathrm{~mm}< \\
y<300 \mathrm{~mm} \text {; grid spacing: } 20 \mathrm{~mm} \text { (along both } \\
X \text { and } Y \text { ); heights: } 25,50,75,100,125 \text { and } 150 \\
\mathrm{~mm}\end{array}$ \\
\hline LDA & $\begin{array}{l}U-W \text { components measure- } \\
\text { ments }\end{array}$ & $\begin{array}{l}\text { Grid: }-300 \mathrm{~mm}<x<300 \mathrm{~mm} ;-300 \mathrm{~mm}< \\
y<300 \mathrm{~mm} \text {; grid spacing: } 20 \mathrm{~mm} \text { (along both } \\
X \text { and } Y \text { ); heights: } 25,50,75,100,125 \text { and } 150 \\
\mathrm{~mm}\end{array}$ \\
\hline LDA & $\begin{array}{l}V-W \text { components measure- } \\
\text { ments }\end{array}$ & $\begin{array}{l}\text { Grid: }-300 \mathrm{~mm}<x<300 \mathrm{~mm} ;-300 \mathrm{~mm}< \\
y<300 \mathrm{~mm} \text {; grid spacing: } 20 \mathrm{~mm} \text { (along both } \\
X \text { and } Y \text { ); heights: } 25,50,75,100,125 \text { and } 150 \\
\mathrm{~mm}\end{array}$ \\
\hline LDA & $\begin{array}{l}\text { Vertical profiles ( } 3 \text { minutes } \\
\text { averages) }\end{array}$ & $\begin{array}{l}14 \text { profiles; heights: } 25,37.5,50,62.5,75,100 \text {, } \\
125,150,175,200,225,250,275,300,325 \text { and } \\
350 \mathrm{~mm}\end{array}$ \\
\hline
\end{tabular}


The whole data set, together with full details of the approach flow and site geometry, is available on request for third party use from the authors.

The flow visualisation experiments highlighted the complexity of the case-study. Complex three-dimensional flows were observed, particularly in the 'upwind' sections of the intersection, where three different fluxes (from Gloucester Place, Marylebone Road, and from the above-canopy flow) interacted. This behaviour cannot be correctly reproduced by current stateof-the-art street canyon and urban dispersion models, which have mostly a two-dimensional approach. The analysis of the video records from the flow visualisation experiments pointed out another important characteristic: intermittently, the average flow conditions were strongly perturbed for a limited period of time, with the associated formation of transient recirculations and changes in the flow paths. This effect could lead to a significant modification to the distribution of pollutants at the intersection and hence the components of any pollutant mass flux balance. Therefore, the phenomenon should be further investigated in order to develop reliable dispersion models for similar complex situations.

The LDA measurements confirmed the qualitative analysis performed with the flow visualisation technique, although no information about the intermittent behaviour was obtained. Measurements were performed over the whole intersection area, with a high resolution grid, for all three components of velocity and turbulence field. This allowed the production of a very detailed map of the flow field and the generation of stream traces. In comparison with the classical, ideal street canyon intersection behaviour these maps sow how in this complex but real situation some details of the upwind flow, such as the wakes from two tall towers, play an important role in defining the flow field within the intersection, particularly at roof level. These effects are likely to influence strongly the mass exchange mechanism between the canopy flow and the air aloft, and therefore the distribution of pollutants. The particularly asymmetric geometry of the area studied enhances the interaction of the canopy flow with flow above roof level, unlike the situation with the classic, ideal street canyon flow, where this interaction is weak and models usually consider the two flows separately.

A mass flux balance for the intersection could be attempted using this data set, especially in conjunction with tracer concentration measurements. However, despite the high resolution of the data, the main restriction on doing so is that the lowest measurement points were located at $z=25 \mathrm{~mm}$ (that is $5 \mathrm{~m}$ at full scale). Neglecting the details of the flow field below this height, which are likely to be quite complex, may well introduce significant errors into any flux calculations.

\section{Acknowledgements}

The DAPPLE project has been funded by the Engineering and Physical Sciences Research Council (EPSRC). The work carried out by Matteo Carpentieri during his $\mathrm{PhD}$ thesis has been funded by the University of Florence.

\section{References}

Arnold SJ, ApSimon H, Barlow J, Belcher S, Bell M, Boddy JW, Britter R, Cheng H, Clark R, Colvile RN, Dimitroulopoulou S, Dobre A, Greally B, Kaur S, Knights A, Lawton T, Makepeace A, Martin D, Neophytou M, Neville S, Nieuwenhuijsen M, Nickless G, Price 
C, Robins A, Shallcross D, Simmonds P, Smalley RJ, Tate J, Tomlin AS, Wang H, Walsh P (2004) Introduction to the DAPPLE Air Pollution Project. Sci Total Environ, 332:139-153

Berkowicz R, Hertel O, Larsen SE, Sørensen NN, Nielsen M (1997) Modelling traffic pollution in streets. Danish Ministry of Environment and Energy, National Environmental Research Institute

Britter RE, Hanna SR (2003) Flow and dispersion in urban areas. Annu Rev Fluid Mech, 35:469-496

Garcia Sagrado AP, Van Beeck J, Rambaud P, Olivari D (2002) Numerical and experimental modelling of pollutant dispersion in a street canyon. J Wind Eng Ind Aerodyn, 90:321-339

Hunt JCR, Carruthers DJ, Britter RE, Daish NC (2002) Dispersion from accidental releases in urban areas. Report ADMLC/2002/3 Atmospheric Dispersion Modelling Liaison Committee

Kastner-Klein P, FedorovichE, Rotach MW (2001) A wind tunnel study of organised and turbulent air motions in urban street canyons. J Wind Eng Ind Aerodyn, 89:849-861

Louka P, Belcher SE, Harrison RG (2000) Coupling between air flow in streets and the welldeveloped boundary layer aloft. Atmos Environ, 34:2613-2621

Meroney RN, Pavageau M, Rafailidis S, Schatzmann M (1996) Study of line source characteristics for 2-D physical modelling of pollutant dispersion in street canyons. J Wind Eng Ind Aerodyn, 62:37-56

Pavageau M, Rafailidis S, Schatzmann M (2001) A comprehensive experimental data-bank for the verification of urban car emission dispersion models. Int J Environ Pollut, 15:417-425

Robins AG, Macdonald RW (2001) Review of flow and dispersion in the vicinity of groups of buildings. ADMLC Annual Report 1998/99, NRPB-R322-AnnexB, Atmospheric Dispersion Modelling Liaison Committee

Scaperdas A-S (2000) Modelling flow and pollutant dispersion at urban canyon intersections. $\mathrm{PhD}$ thesis, Imperial College of Science, Technology and Medicine, University of London

Schatzmann M, Rafailidis S, Pavageau M (1997) Some remarks on the validation of small-scale dispersion models with field and laboratory data. J Wind Eng Ind Aerodyn, 67-68:885-893

Soulhac L (2000) Modélisation de la dispersion atmosphérique á l'intérieur de la canopée urbaine. PhD thesis, École Centrale de Lyon, France

Uehara K, Murakami S, Oikawa S, Wakamatsu S (2000) Wind tunnel experiments on how thermal stratification affects flow in and above urban street canyons. Atmos Environ, 34:15531562

Vardoulakis S, Fisher BEA, Pericleous K, Gonzalez-Flesca N (2003) Modelling air quality in street canyons: a review. Atmos Environ, 37:155-182

Xie X, Huang Z, Wang J, Xie Z (2005) The impact of solar radiation and street layout on pollutant dispersion in street canyon. Atmos Environ, 40: 201-212 
Xie Z-T, Coceal O, Castro I (2008) Large-Eddy simulation of flows over random urban-like obstacles. Boundary-Layer Meteorol, 129:1-23 